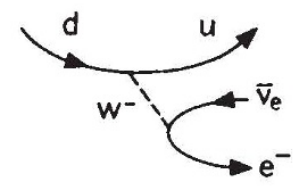

$\beta$ - decay

$\mathrm{d} \longrightarrow \mathrm{ue}^{-} \bar{v}_{\mathrm{e}}$

forward. The first of these is by a collaboration involving Salam (see this issue of Nature page 736) who report in a footnote that Weinberg has also independently worked out a similar idea. In a nutshell: just as the quarks feel the strong interaction but leptons do not, so the 'symmetry' is restored by postulating that leptons feel a new interaction which the quarks do not. This is the origin of the excess of the (purely leptonic) Gargamelle events.

\section{Leptons, quarks and colour}

The salient features of the original Weinberg-Salam model were as follows. The elementary particles are of two types-leptons and quarks. Both leptons and quarks experience the electromagnetic and weak forces. The quarks but not the leptons can also experience the strong (nuclear) force.

In the phenomena experienced in our everyday world wc meet just two leptons-the electron in atoms and its ghostly brother the neutrino in radioactivity and the burning of the Sun, and two flavours of quark-up and down-which build up the neutron, proton and ultimately nuclei. These quarks and leptons form the 'first generation of elementary fermions'.

In the weak interaction of radioactivity it has been known for many years that the neutrino turns into an electron or that an up quark transmutes into a down. The process of $\beta$ decay is the best known example (see figure). The electrical charge is changed at the lepton vertex but is exactly balanced by an equal but oppositc change at the quark vertex. This is an example of a 'charge changing weak interaction'.

A prediction of the Weinberg-Salam model was that neutral weak interactions should also exist where a neutrino stays a neutrino and an up (or down) quark remains an up (down) quark. Such an interaction was subsequently found (Nature 245, 119; 1973; 249, 211; 250, 186; 1974) with properties consistent with the predictions of the model.

There will also be a weak neutral current where an electron turns into an electron. This will be additional to the familiar electromagnetic current of the electron. The latter is most familiar in atomic physics. The presence of the weak neutral current will give rise to interesting new phenomena such as parity violation in atomic physics (see News and Views 264, 505; 1976 and

\title{
Virion RNA can be used to study nuclear processing of messenger RNA
}

\author{
from $R$. Weiss
}

WrTH the discovery of splicing of mRNA in eukaryotes there has been renewed interest and excitement in the nuclear processing of mRNA from larger transcripts. One problem in studying the editing mechanisms of mRNA production is the difficulty of obtaining substantial amounts of stable and pure precursor RNA for use in processing experiments. On page 779 of this issue of Nature, Stacey and Hanafusa describe a promising system for such studies. The $35 \mathrm{~S}$ genomic subunits of virion RNA of avian RNA tumour viruses resemble mRNA in structure and can be translated in vitro to synthesise the product, Pr76, of the gag gene which is situated at the $5^{\prime}$ end of the RNA molecule. The env gene, coding for virion envelope glycoproteins is located nearer the $3^{\prime}$ end of the $35 \mathrm{~S}$ genomic RNA and is not translated efficiently in vitro. In the cytoplasm of infeoted cells, however, a $21 \mathrm{~S}$ mRNA has been isolated which carries the genetic sequences of the env gene (Hayward J. Virol. 24, 47; 1977). The subgenomic $21 \mathrm{~S}$ mRNA seems to be a spliced message as it includes a $5^{\prime}$ terminal leader sequence juxtaposed with sequences derived from the $3^{\prime}$ half of the virion molecule (Mellon \& Duesberg Nature 270, 631; 1977; Weiss, Varmus \& Bishop Cell 12, 983; 1977).

Stacey and Hanafusa have used a sensitive means of detecting synthesis of small amounts of envelope glycoprotein translated from the $35 \mathrm{~S}$ genomic RNA of an avian leukosis virus, RAV-2, microinjected into cells. The cells used for microinjection are already infected with a strain of Rous sarcoma virus (RSV) that is defective in envelope glycoprotein. If glycoprotein is synthesised by translation of the RAV-2 RNA, the defective RSV is complemented and infectious RSV particles are produced. Using this technique theiy show that $35 \mathrm{~S}$ genomic RNA inoculated directly into the nucleus results in rescue of the defective RSV, whereas inoculation of the same RNA preparation into the cytoplasm

the figure in News and Views 273, 99; 1978).

It seems that to make the model fully self-consistent theoretically at extremely high energies, the total amount of electrical charge of the elementary fermions must be zero. The leptons have a total of -1 (due to the barely yields any infectious RSV. As a positive control, cytoplasmic inoculation of $21 \mathrm{~S}$ message causes the most efficient RSV rescue. Stacey and $\mathrm{Ha}$ nafusa have not been able to follow the fate of the microinjected RNA directly, but on the basis of these data, it seems that nuclear processing of the inoculated virion RNA takes place, yielding an efficient env messenger molecule which is then transported to the cytoplasm and is translated into the glycoprotein precursor. The supposition is that the natural $21 \mathrm{~S}$ env mRNA is produced by the same mechanism, rather than by a direct transcription of subgenomic mRNA from the DNA provirus. Nevertheless, a considerable amount of 35S RNA is exported intact to the cytoplasm to serve as mRNA for gag proteins and polymerase as well as for genomic RNA to be packaged into virions. The most important result of the evidence for nuclear processing is, as the authors note, that virion RNA can be used as a readily obtainable 'nuclear' precursor for mRNA. This, one hopes, will open the way for testing mRNA processing in vitro using nuclear extracts which may lead to the identification of splicing enzymes.

It should be pointed out, however, that in a previous study using the same RSV rescue technique, Murphy and Ozanne (in Avian RNA tumor viruses, (eds Barlatti \& de GiuliMorghen) Piccin Medical Books, Padua, 1978) showed that 35 S genomic RNA introduced into cells in culture by treament with DEAE. dextran resulted in glycoprotein production (as well as gag proteins) almost as efficiently as Stacey and Hanafusa obtained by nuclear microinjection. Again, the fate of the 'transfected' RNA could not be followed directly, but the discrepancy in translation of virion RNA introduced into the cytoplasm by microinjection or by transfection requires explanation.

R. Weiss norks at the Imperial Cancer Research Fund Laboratories, London.

electron; the neutrino is electrically neutral). The up and down quarks have charges of $\frac{2}{3}$ and $-\frac{1}{3}$ respectively yielding a total quark charge of only $\frac{1}{3}$ whereas a net +1 is needed to compensate for the leptons net -1 . Fortunately there is evidence from various independent data that suggest 